\title{
The Inflammatory Processes Driven by Gut Microbiota
}

Ayşe Buruş ${ }^{1}$

ORCID: 0000-0002-9464-7053

Başak Çeltikçi', [MD]

ORCID: 0000-0002-3242-978X

Yasemin Aksoy', [MD]

ORCID: 0000-0001-5034-2779

\section{nee) ABSTRACT Cen}

Microbiome studies have shown alterations in bacterial communities in the state of many diseases, including inflammatory bowel disease, metabolic disorders, autoimmune diseases, neurodegenerative diseases, and cancer. Chronic inflammation is a common promoter of many of these pathological processes. Shifting in the microbial diversity is also known as dysbiosis. Dysbiosis, increased detrimental bacterial products, decreased favorable microbial metabolites, interrupted tissue barriers, and bacterial translocation cause excessive immune response and inflammation. Several mechanisms play a role to maintain intestinal homeostasis by limiting bacterial translocation from the intestinal lumen into the lamina propria. Among these mechanisms, most importantly, the mucosal barrier that consists of the antimicrobial peptides, mucus, and immunoglobulin A is fundamental to protect epithelial barrier integrity to reduce the excessive immune response. Moreover, recognizing bacteria and metabolites through receptors results in $\mathrm{T}$ cell regulation and immune modulation, which is the keystone of the controlled immune response. This review summarizes the anti-inflammatory and pro-inflammatory mechanisms driven by gastrointestinal microbiota, and it also highlights the recent approaches, including epigenetics and precision medicine.

Keywords: Gastrointestinal microbiome; host microbial interactions; inflammation; immune system; precision medicine.

https://doi.org/10.32552/2021.ActaMedica.548

Received: 21 December 2020, Accepted: 5 August 2021,

Published online: 13 August 2021

\section{INTRODUCTION}

Human microbiota include a diverse microbial community that is composed of archaea, viruses, fungi, protozoa but also mainly bacteria [1]. As a great number and variety of bacteria inhabiting the human gastrointestinal tract through a lifetime, gut microbiota may be counted as such a chronic environmental factor on human health. Due to the fact that intestinal microbiota experiments have been gaining attention over the years, specifically with the developments in Metagenomics, such as the advancement of next-generation sequencing (NGS) technologies, including $16 \mathrm{~S}$ ribosomal RNA (16S rRNA) sequencing, supported researchers with the ability to investigate the structure of diverse microbial species and characterize their composition to understand the complex microbial ecology of intestinal tract $[2,3]$. In parallel, the
Human Microbiome Project was initiated by the United States National Institutes of Health to classify and describe human microbial flora [4].

Microbiome experiments are not only designed to detect the taxonomic diversity of the microbiome, but also to consider their different effects on the host physiology and pathology of different diseases [4]. Since a large number of studies have shown substantial connections between various microbes and a variety of diseases, including inflammatory bowel disease (IBD), metabolic disorders, autoimmune diseases, neurodegenerative diseases, and cancer, a large number of review articles investigate the relationship between gut microbiota and these diseases [5-9]. These findings suggest that chronic inflammation is a promoter of 
many of these diseases, although inflammation is a natural physiological response of the body to the infiltration of foreign pathogens, and it provides the required defensive response and facilitates the healing process for injured tissue $[10,11]$.

It is stated that Firmicutes, Bacteroidetes, Proteobacteria, and Actinobacteria are the major bacterial phyla in the human intestine. Firmicutes and Actinobacteria phyla are gram-positive bacterias that include, respectively, lactic acid bacteria and Bifidobacteria, which are known as probiotics. On the other hand, Bacteroidetes and Proteobacteria phyla are Gram-negative bacteria that are pathogenic with lipopolysaccharides (LPS) on their outer membrane, therefore, they can play a role in infection and inflammatory processes $[12,13]$. Due to the gain or loss of certain bacteria or changes in the relative abundance of microbes, there can be an imbalance in the gut microbial community, which is defined as dysbiosis. Dysbiosis is not only shifting in the composition of microbiota, but also an alteration in their functions [14]. The occurrence of dysbiosis can lead to increased barrier permeability and increased inflammatory cytokines resulting in inflammation [15].

Probiotics, prebiotics, postbiotics, and parabiotics are potential treatment methods in modulation microbiota as well as antibiotics. Probiotics are living microorganisms, which their administration in adequate amounts provides beneficial effects on health [16]. Prebiotics, including inulin derivates, are nutrients for the probiotics so their role is to promote the beneficial bacteria composition. The recent approach in microbial therapy focuses on postbiotics and parabiotics. While the parabiotics are the inactivated cells of probiotics, postbiotics are the cell-free supernatants, which include a mixture of metabolites secreted by probiotics, such as short-chained fatty acids (SCFAs), enzymes, secreted proteins, peptides, amino acids, and vitamins [17].

Since the inflammatory processes of the host are modulated by the gut microbiota and metabolites, to understand those interactions is fundamental to devise new prevention and treatment approaches. In this review, we summarize the mechanisms through which the intestinal microbiome plays an important role in maintaining homeostasis or inducing inflammation.

\section{Intestinal Homeostasis: A Balance Between Physiological and Chronic Inflammation}

The intestinal tract is one of the most complex areas in the human body in which a great number and variety of microorganisms inhabit. For this reason, the interactions with the microbiome and intestinal epithelial cells are inevitable. Exposure of these epithelial cells to a variety of factors, such as pathogenic bacteria, several toxic components, bacteria-derived metabolites, or other dietary antigens, can induce the immune system and inflammatory response of the host [18].

As we mentioned above before, inflammation is a protective response to tissue injury or infection by the immune system. Hence, an appropriate inflammatory response to pathogens is necessary for neutralizing their detrimental effects. On the other hand, this response to commensal bacteria should be limited as the chronic inflammation is related to several diseases, such as IBD. In other words, to establish a tolerance for beneficial microbiota and to evoke an immune response to gut pathogens is essential [10].

It is the fact that controlled immune responses are needed to maintain this critical balance between protective inflammatory and detrimental inflammatory processes. Thus, several mechanisms play a role to maintain intestinal homeostasis, which enables the control of inflammation by limiting bacterial translocation from the intestinal lumen into the lamina propria and other extraintestinal sites through the mucosal and epithelial barriers. Other immune components and signaling pathways will be explained in further sections. The intestinal environment during homeostasis is illustrated in Figure 1.

\section{Importance of the Mucosal and Epithelial Barriers to Maintain Intestinal Homeostasis}

Luminal gut microbiota and intraintestinal immune compartments are segregated by intestinal epithelial cells, which provide a physical barrier. There is also a mucosal layer that ensures a biochemical barrier. Initially, the mucus layer provides protection for host tissue from microbiota by restricting the contact, limiting bacterial exposure, and then reducing the inflammatory response [19]. 
Various studies using animal models have clearly shown that disruption of epithelial or mucosal barriers and then bacterial translocalization are keystones in the pathogenesis of inflammation in the gut $[19,20]$.

Intestinal mucosa consists of the antimicrobial peptides, mucus, and immunoglobulin A ( $\lg A)$ secreted or produced by goblet cells, epithelial cell lineages, and dendritic cells, respectively. Antimicrobial peptides, such as a-defensin and Reg3y, are not only a component of the mucosal layer but also modulators to shape the composition of the microbiota. For example, a-defensin has antimicrobial properties like disrupting bacterial cell walls or the inner membrane by enzymatic attack. Secreted IgA also modulates the bacterial composition as well as antimicrobial peptides [21].

Similar to antimicrobial peptides secreted by host cells, some bacterial strains are also capable of producing antimicrobial peptides, such as bacteriosins [22]. Due to their antimicrobial properties, they ensure resistance to pathogen invasion to the mucus and epithelial layer.

Moreover, SCFA have also a major role in maintaining the integrity of the mucus layer, because of its properties, such as being a significant energy source for the colonic epithelium and maintaining tissue integrity [23].

\section{How to Recognize the Bacteria and Their Metabolites in the Intestine}

The host requires defense mechanisms to protect itself from pathogenic bacteria. Initially, pattern recognition receptors (PRR) act as a detector of certain molecules that are related to pathogenic bacteria. These molecules are referred to as pathogen-associated molecular patterns (PAMPs) that contain bacterial peptides, such as flagellin, bacterial nucleic acids, peptidoglycans, and bacterial carbohydrates, especially (LPS). Second, PRRs not only recognize these PAMPs, but also initiate the immune response and mediate cytokine release [24].

The two major PPRs subgroups receptor for recognizing the bacteria in the intestine are Tolllike receptors (TLRs) that are membrane-bound and NOD-like receptors (NLRs), which take place in the cytoplasm. NLRs lead to the formation of multiprotein complexes that are called inflammasomes.
When inflammasomes are induced by bacterial pathogens, they activate caspase- 1 , and then it results in the secretion of proinflammatory cytokines, such as interleukin-1 $\beta$ (IL-1 $\beta$ ) [25]. Besides, to recognize pathogens, inflammasomes also have the ability to sense commensal bacteria and their metabolites, thus emphasizing the active role of inflammasomes in host-microbiota interactions by sensing and responding to commensal and pathogenic bacteria [25].

\section{Immune Modulation and Maintaining the Homeostasis Through Intestinal Microbiota}

Many studies with germ-free mice provide a striking illustration of how microbiota can have an important effect on the immune modulation. As no microbial community inhabiting their intestine, a number of studies reveal that the T-cell differentiation is impaired in the germ-free mice intestine, which causes several immune defects [26]. Hapfelmeier et al. demonstrate that IgA production is decreased in these mice [27]. Interestingly, recolonization of these mice via the administration of fecal microbiota can reverse many of these disruptions $[28,29]$.

$\lg$ A secretion is one of the keystones in intestinal homeostasis. When the bacteria sensed through TLRs and NLRs, it results in the production of IgAsecreting $B$ cell and Treg differentiation in healthy subjects, so $\lg A$ can neutralize the pathogenic bacteria. Wilmore et al. demonstrate that naive mice in which Proteobacteria transferred, represent an increase in IgA levels in their serum, so that highlights the composition of intestinal microbiota and selected bacteria are involved in the serum $\lg \mathrm{A}$ concentration. Besides, following intestinal barrier dysfunction, serum IgA protects against lethal sepsis [21].

Another mechanism that inhibits intestinal inflammation is IL-10 production. IL-10 is an immunosuppressive cytokine, which is able to inhibits microbiota-driven proinflammatory cytokine release. Wu et al. showed that IL10 producing $B$ cells in the intestine suppress excessive immune responses and sustain mucosal homeostasis [30]. Most studies underline that Treg-cells (Th1) which subsets of CD4+ T cell, produces IL-10 and provides mucosal homeostasis [31]. Several studies present that the regulation of intestinal homeostasis via IL-10 plays a significant 
role in IBD prevention [31]. It is also known that resident intestinal microbiota enhances IL-10 production by an increase in IL-10 producing $B$ cells and subsets of CD4+ T cells [32]. All these studies demonstrated that IL-10 is one of the most important anti-inflammatory cytokines and its production is inducible by commensal bacteria. The anti-inflammatory processes thus far are summarized in Figure 1.

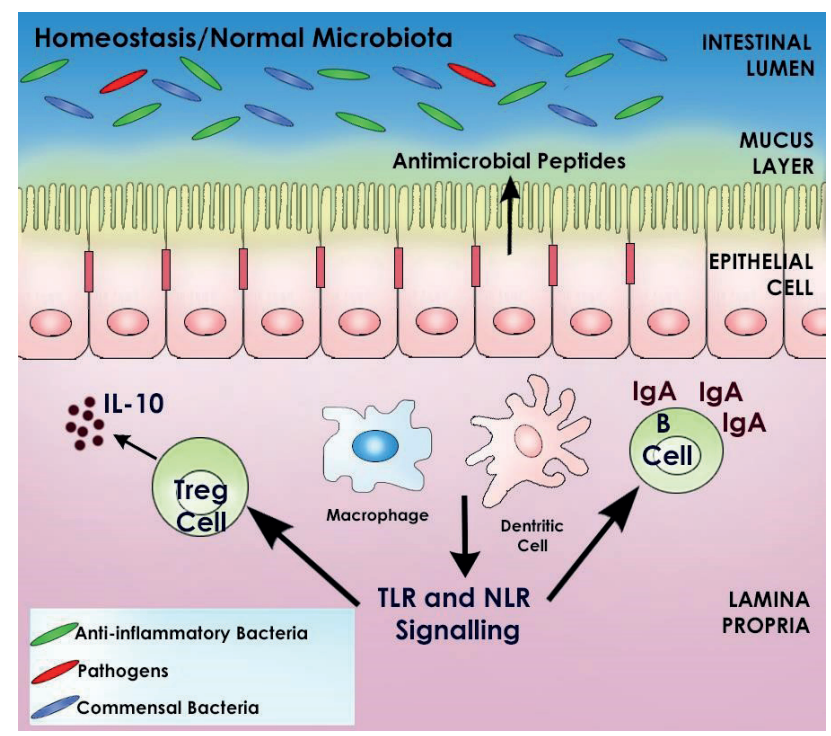

Figure 1. Overview of intestinal homeostasis: The mucus layer contains antimicrobial peptides and protects the epithelial cells from bacterial translocation. The CD4+ regulatory $T$ cells (Treg) and B cells prevent inflammation through the release of anti-inflammatory cytokine IL-10 and IgA, respectively, via TLR and NLR signaling.

It is the fact that an enormous quantity of molecules is produced by intestinal microbiota in interaction with the host. Various studies have shown that many bacterial species have protective properties against inflammation through the variety of metabolites they produce. The most wellknown beneficial bacterial metabolites are SCFAs, such as butyrate, acetate, propionate, produced by bacterial fermentation of dietary fiber and resistant to starch [23]. Faecalibacterium prausnitzii from the phylum Firmicutes and Clostridium leptum from the family Ruminococcaceae are the most known member of the butyrate-producing bacteria. Similarly, Bifidobacterium species from the phylum Actinobacteria produce acetate and lactate via carbohydrate fermentation [23].

As well as SCFAs have been an important energy source for epithelial cells to maintain barrier integrity, they are also considered as significant anti-inflammatory bacterial products, since many studies reveal that regulatory T-cell proliferation is stimulated by SCFAs directly. In addition to the regulation of T-cell differentiation, SCFAs promotes the IgA-producing B-cells by activating $\mathrm{G}$ proteincoupled receptor 43 (GPR43) [33]. Another inflammation suppressor property of SCFAs is promoting IL-10 production in the gut [34].

As mentioned formerly, the production of the antimicrobial peptide is a significant part of maintaining homeostasis by facilitating the mucus and epithelial barrier function. Zhao et al. indicate that in Gpr43 Knockout Mouse has impaired AMPs like Regllly and $\beta$-defensins expression. On the other hand, the activation of Gpr43 by microbiotaproduced butyrate induces AMP production in both in vitro and in vivo models [33].

Microbiota-produced metabolites, such as polysaccharide A (PSA), which produced by Bacteroides Fragilis, have also been caused an increase of IL-10-producing CD4+ $\mathrm{T}$ cells and Treg cell accumulation that result in resistance to pathogenic bacterial colonization [35]. Hence, PSA expression is crucial to suppress excessive IL17 production and for anti-inflammatory defense [32,34].

Moreover, an anti-inflammatory protein, the microbial anti-inflammatory molecule (MAM), is derived from the commensal bacteria Faecalibacterium prausnitzii [36]. The MAM protein has been shown to prevent dinitrobenzene sulfonic acid-induced colitis in mice by inhibition of the NF$K B$ pathway in epithelial cells [37]. Recently, Xu et al. demonstrated that MAM protein can restore the intestinal barrier function by regulating the tight junction pathways in the diabetic intestinal epithelial cells line [38].

\section{Dysbiosis and Chronic Inflammation}

As we mentioned before, the dominant gut bacterial composition of healthy individuals consists of Firmicutes and Bacteroidetes, followed by Proteobacteria and Actinobacteria [39]. Several clinical trials have shown that changes in bacterial variation, especially alteration in the ratio of Firmicutes to Bacteroidetes, enable to trigger inflammation [40]. These studies emphasize the balance among bacterial species is critical for regulating inflammation and disease progression. 
Those alterations in microbial diversity and their consequences as an imbalance among the microbial species is referred to as dysbiosis, which takes a role in disrupting host-microbiota homeostasis [40]. In healthy individuals, intestinal bacteria exhibit homeostatic balance in both commensal and potential pathogens, which prevents pathogenic bacterial overgrowth via colonization resistance. Once microbiota is altered, certain pathogens can take advantage, furthermore, even they can cause dysbiosis themselves [40].

Dysbiosis occurs due to the loss of beneficial bacteria, excessive growth of pathogenic bacteria, loss in total diversity of bacteria or, a combination of these phenomena [41]. There are several factors that have been shown in various studies that can cause dysbiosis, including both environmental factors and (epi)genetic factors [42]. Diet and especially the use of medications, such as antibiotics, participate in the onset of dysbiosis, just as it is caused by stress and infections $[42,43]$. Drivers of dysbiosis are illustrated in Figure 2.

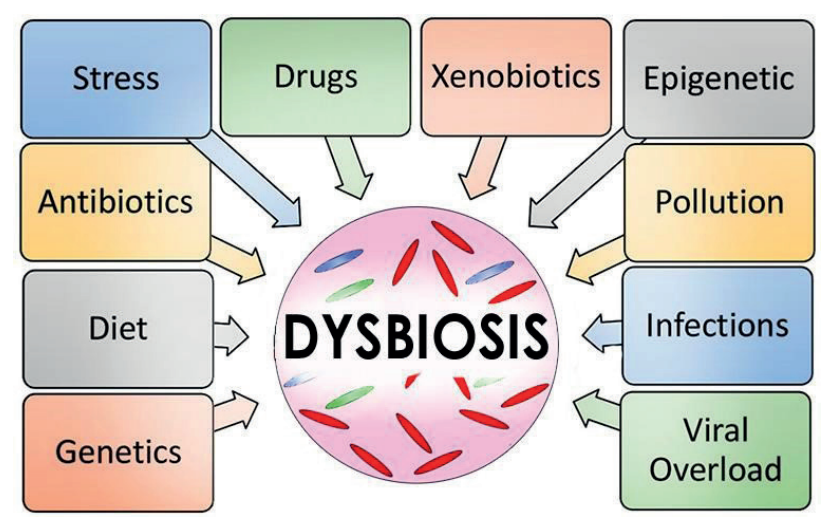

Figure 2. Overview of dysbiosis drivers: Both environmental and genetic factors display in the pathology of dysbiosis.

Dysfunction in the regulation of host-intestinal microbiota homeostasis, that is caused by dysbiosis, can result in an excessive immune response to the intestinal microbiota, which results in chronic inflammation. This mechanism participates in both the initiation and progression of IBD, and it is also essential in the prognosis of this disease [44]. A large number of studies highlight that an intact epithelial barrier is vital to preserve the homeostatic tolerance, as the breakdown of the mucosal and epithelial layers of organs is the common aspect of microbiota-driven inflammatory diseases [45]. The entry of bacteria and their metabolites to compartments, via depleted mucus layer and disrupted epithelial cells, induce a chronic inflammatory response as resident immune cells are exposed to a large number of bacterial antigens. The overall excessive inflammatory response due to dysbiosis is schematized in Figure 3.

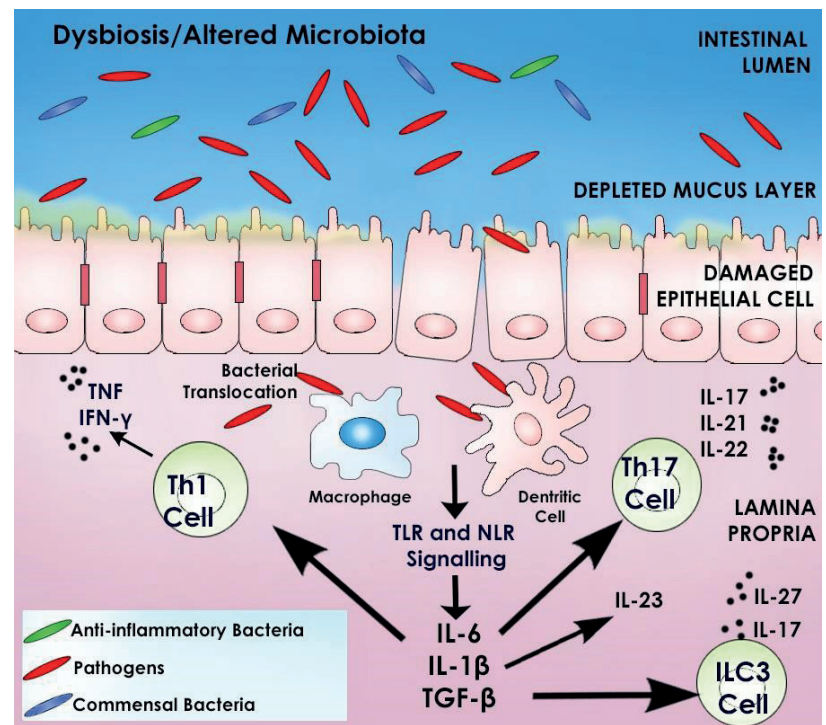

Figure 3. Overview of inflammatory response in the intestine: Macrophages, dendritic cells, and epithelial cells interact with altered microbiota and their metabolites via TLR and NLR signaling. The consequence of the depleted mucus layer and damaged epithelial cells by dysbiosis is the differentiation of type ILC3s, Th17 cells, and Th1 cells, which participate in excessive secretion of proinflammatory cytokines and sustain inflammation.

The excessive immune response via Th1 and Th17 cells against certain bacteria has been remarked in a dysbiotic state. Th1 and Th17 cells are able to produce pro-inflammatory cytokines, such as tumor necrosis factor (TNF), so that they lead to tissue injury and then exacerbate the exposure to bacterial compounds by TLRs. The role of TNF as a proinflammatory cytokine is to stimulate the secretion of other proinflammatory cytokines, including IL-2 and IL-6, which promote chronic inflammation $[46,47]$.

Another important cytokine is IL-6, which is capable of the feature proinflammatory properties and contributes to T cell survival. Jeon et al. reported that IL-6 gene expression in basophils is stimulated by bacterial protein flagellin through TLR5 [48]. Bacterial flagellin is not only a ligand for TLR5, but also triggers the NLRC4 inflammasome, which results in the activation of caspase- 1 and then 
persistent IL-1 $\beta$ cytokine secretion [49]. Shikama et al. revealed that IL-1 $\beta$ activation in tissues can be triggered by Muramyl dipeptide (MDP), which is a compound of the bacterial cell wall and is mentioned as a PAMP recognized through PRRs [50]. Therefore, excessive IL-1 $\beta$ secretion induces other proinflammatory cytokines, such as IL-17, by promoting Th17 cell differentiation $[51,52]$.

Group 3 innate lymphoid cells (ILC3) secrete cytokines as a response to inflammation and microbial infections, such as caused by Citrobacter rodentium [53]. Although the ILC3s participate in intestinal homeostasis under physiological conditions, the dysregulation of them leads to excessive inflammatory cytokines expression, such as IL-17. Overexpression of these cytokines brings along the disruption of E-cadherin and epithelial cell junction, which results in increased epithelial cell permeability [54]. Due to the increased epithelial cell permeability, bacteria associated molecules infiltrate into the circulation, that means microbiota not only participates in local inflammation, but also able to induce inflammation in distant sites. Correspondingly, the LPS, which is a significant fragment of the Gram-negative bacterial cell wall, has been shown with high serum levels in several studies on adipose tissue inflammation [55].

Taken together, the overall knowledge has represented that dysbiosis, increased detrimental bacterial products, decreased favorable microbial metabolites, interrupted tissue barriers, and bacterial translocation cause excessive immune response and inflammation.

\section{Microbiota-related Inflammation in IBD}

Many various studies have reported alterations in the composition of gut microbiota in addition to increased gut permeability in IBD. Those alterations reveal a decrease in the number of antiinflammatory bacteria [56]. The changes in the bacterial composition in patients with IBD shown in Figure 4.

Macrophages, dendritic cells, and epithelial cells interact with altered microbiota and their metabolites via TLR and NLR signaling. The depleted mucus layer and damaged epithelial cells by dysbiosis, leads to differentiation of type ILC3s, Th17 cells, and Th1 cells, which participate in excessive secretion of proinflammatory cytokines and sustain inflammation.

\section{THE BACTERIAL COMPOSITION CHANGES IN PATIENTS WITH IBD}

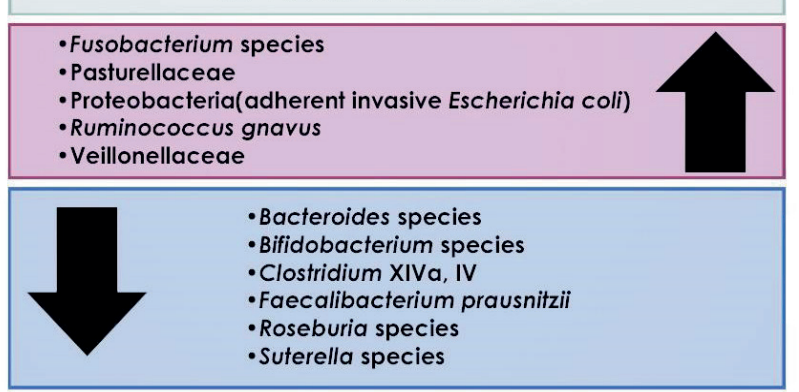

Figure 4. The overall alterations of bacterial composition in patients with IBD.

Regarding to this statement, Zhu et al. remark the editing of the microbiota composition by various treatments improves the adverse effects of dysbiosis in a state of intestinal inflammation [57]. However, it is still unclear whether microbiota alterations in IBD are the cause or the result [58].

\section{Microbiota-induced Inflammation as a Cancer Driver}

Pathogens also play a crucial role in the pathology of several diseases by inducing chronic inflammation, in addition to alterations in the variety of commensal bacteria. As chronic inflammation is one of the ultimate drivers of certain cancers, especially colorectal cancer (CRC), a variety of pathogenic bacteria takes a role in promoting tumorigenesis in CRC. Specifically, the bacteria that have the ability to attach epithelial cells, such as Helicobacter pylori, Enterococcus spp, few members of the Enterobacteriaceae family, and some strains of Escherichia coli like Streptococcus bovis. All of these bacteria can trigger epithelial cell proliferation directly, which results in hyperplasia. Due to the toxins they produce, they can distort the epithelial barrier integrity, then cause cell damage and induce inflammation [44].

Grivennikov et al. indicated that decreased mucin secretion and suppressed E-cadherin expression cause damaged epithelial cell integrity in CRC. This leads to excessive Th17 cell immune response, which also triggers IL-23 secretion. Its secretion will be followed by IL-6, IL-17, and IL-22 cytokine production that result in aggravating both tumor initiation and progression [59]. Altogether, this data displays the crucial role of microbiota in stimulating pro-tumorigenic inflammation. 


\section{Inflammation and Possible Role of Epigenetic Alterations by Microbiota}

The relationship between epigenetic marks (DNA methylation and histone modification) and the gut microbial community has not been clarified completely thus far. Nevertheless, recent perspectives have proposed that epigenetic regulation by gut microbiota may participate in inflammatory processes. This notion is based on the mechanism that microbial metabolites are able to affect the activity of the enzymes, which regulate DNA methylation and histone acetylation or deacetylation [60].

Recently, Corte et al. indicated that microbiota derived SCFAs, butyrate and propionate, contribute to regulating several epigenetic enzymes in chromatin model alteration, such as histone acetylation. Moreover, it is shown that both butyrate and propionate reduce IL-1 $\beta$ secretion by inhibiting the transcription of the IL1 $\beta$ gene in macrophages [61].

Consequently, histone deacetylase (HDAC) inhibition by SCFAs is highlighted as the major component in the regulation of inflammation through many epigenetic studies [40]. Yet, this area is elusive and still unilluminated enough; therefore, epigenetic mechanisms need to be extensively studied on the basis of host-microbiota interaction.

\section{CONCLUSION}

This review covers the overall knowledge about intestinal microbiota associated with inflammatory mechanisms. These mechanisms are maintaining intestinal homeostasis, recognizing bacteria and bacteria-derived molecules, responding to those bacteria associated molecules, and antiinflammatory \& pro-inflammatory processes subsequently, thus far.

Thanks to the advance of novel methods, especially NGS/16S rRNA sequencing, enables us to probe the bacterial diversity in the gut. In this review, we demonstrate that there are various microbial alterations occur in several inflammatory diseases, such as IBD, autoimmune diseases, cancer, metabolic diseases, and neurological disorders. These alterations in the microbial community of these diseases, include both the increase in pathogen bacteria and decrease in beneficial bacteria (e.g., butyrate-producing bacteria). Understanding the underlying mechanisms of inflammation related to microbiota alterations has become essential to reveal the pathophysiology of these complex inflammatory diseases and their precise treatment with anti/pro/para/postbiotics or fecal transplantation to maintain intestinal homeostasis.

Although several anti-inflammatory and proinflammatory mechanisms between the host and microbiota are enlightened by current data, there is still unmet need for comprehensive research to clarify the exact cause and effect relationship in complicated inflammatory processes. Inducing inflammation may be both beneficial and detrimental due to the precise balance between necessary or excessive inflammatory responses, and vital in the personalized treatment of inflammatory diseases.

Therefore, these treatment methods by using microbiota modulation still do not have consistent results due to the complex intestinal environment, in which various microbial metabolites, genomes, and the variants of genomes exist together. Treating patients with inflammatory and metabolic diseases must be personalized. To discover all these inflammation related interactions and microbiotaassociated pathways, such as the epigenetic modulation mechanisms, and considering them in the same context together, will enable us to understand and devise novel treatment methods as well as the diagnostic and preventive approaches to these inflammatory and metabolic diseases.

\section{CONFLICT Of INTEREST STATEMENT}

The authors declare they have no conflicts of interest. 
[1] Ursell LK, Metcalf JL, Parfrey LW, Knight R. Defining the human microbiome. Nutr Rev. 2012; 70(1): 38-44.

[2] Pasolli E, Asnicar F, Manara S, et al. Extensive Unexplored Human Microbiome Diversity Revealed by Over 150,000 Genomes from Metagenomes Spanning Age, Geography, and Lifestyle. Cell. 2019; 176(3): 649-662.

[3] Tang L. In vitro intestine model for gut microbiome. Nat Methods. 2019;16(7):578.

[4] Integrative HMPRNC. The Integrative Human Microbiome Project. Nature. 2019; 569(7758): 641-648.

[5] Berer K, Mues M, Koutrolos M, et al. Commensal microbiota and myelin autoantigen cooperate to trigger autoimmune demyelination. Nature. 2011; 479(7374): 538-541.

[6] Clavel T, Desmarchelier C, Haller D, et al. Intestinal microbiota in metabolic diseases: from bacterial community structure and functions to species of pathophysiological relevance. Gut Microbes. 2014; 5(4): 544-551.

[7] Glassner KL, Abraham BP, Quigley EMM. The microbiome and inflammatory bowel disease. J Allergy Clin Immunol. 2020; 145(1): 16-27.

[8] Vivarelli S, Salemi R, Candido S, et al. Gut Microbiota and Cancer: From Pathogenesis to Therapy. Cancers (Basel). 2019; 11(1): 38.

[9] Glass CK, Saijo K, Winner B, et al. Mechanisms underlying inflammation in neurodegeneration. Cell. 2010; 140(6): 918-934.

[10] Kemp WL, Burns DK, Brown TG. 1st Ed. Inflammation and Repair. In: Pathology: The Big Picture. New York, NY; The McGraw-Hill Companies, 2008: 13-22.

[11] Blander JM, Longman RS, lliev ID, Sonnenberg GF, et al. Regulation of inflammation by microbiota interactions with the host. Nat Immunol. 2017; 18(8): 851-860.

[12] Jandhyala SM, Talukdar R, Subramanyam C, et al. Role of the normal gut microbiota. World J Gastroenterol. 2015; 21(29): 8787-8803.

[13] Tap J, Mondot S, Levenez F, et al. Towards the human intestinal microbiota phylogenetic core. Environ Microbiol. 2009; 11(10): 2574-2584.

[14] Kriss M, Hazleton KZ, Nusbacher NM, et al. Low diversity gut microbiota dysbiosis: drivers, functional implications and recovery. Curr Opin Microbiol. 2018; (44): 34-40.

[15] Farre R, Fiorani M, Abdu Rahiman S, et al. Intestinal Permeability, Inflammation and the Role of Nutrients. Nutrients. 2020; 12(4): 1185.

[16] Hill C, Guarner F, Reid G, et al. Expert consensus document. The International Scientific Association for Probiotics and Prebiotics consensus statement on the scope and appropriate use of the term probiotic. Nat Rev Gastroenterol Hepatol. 2014; 11(8): 506-514.

[17] Tsilingiri K, Barbosa T, Penna G, et al. Probiotic and postbiotic activity in health and disease: comparison on a novel polarised ex-vivo organ culture model. Gut. 2012; 61(7): 1007-1015.
[18] Kogut MH, Lee A, Santin E. Microbiome and pathogen interaction with the immune system. Poult Sci. 2020; 99(4): 1906-1913.

[19] Pastorelli L, De Salvo C, Mercado JR, et al. Central role of the gut epithelial barrier in the pathogenesis of chronic intestinal inflammation: lessons learned from animal models and human genetics. Front Immunol. 2013; (4): 280.

[20] Marchiando AM, Shen L, Graham WV, et al. The epithelial barrier is maintained by in vivo tight junction expansion during pathologic intestinal epithelial shedding. Gastroenterology. 2011; 140(4): 1208-1218.

[21] Wilmore JR, Gaudette BT, Gomez Atria D, et al. Commensal Microbes Induce Serum IgA Responses that Protect against Polymicrobial Sepsis. Cell Host Microbe. 2018; 23(3): 302-311.

[22] Simons A, Alhanout K, Duval RE. Bacteriocins, Antimicrobial Peptides from Bacterial Origin: Overview of Their Biology and Their Impact against Multidrug-Resistant Bacteria. Microorganisms. 2020; 8(5): 639.

[23] Parada Venegas D, De la Fuente MK, Landskron G, et al. Short Chain Fatty Acids (SCFAs)-Mediated Gut Epithelial and Immune Regulation and Its Relevance for Inflammatory Bowel Diseases. Front Immunol. 2019; (10): 277.

[24] Kumar H, Kawai T, Akira S. Pathogen recognition by the innate immune system. Int Rev Immunol. 2011; 30(1): 1634.

[25] Franchi L, Munoz-Planillo R, Nunez G. Sensing and reacting to microbes through the inflammasomes. Nat Immunol. 2012; 13(4): 325-332.

[26] Tanoue T, Morita S, Plichta DR, et al. A defined commensal consortium elicits CD8 T cells and anti-cancer immunity. Nature. 2019; 565(7741): 600-605.

[27] Hapfelmeier S, Lawson MA, Slack E, et al. Reversible microbial colonization of germ-free mice reveals the dynamics of $\lg$ A immune responses. Science. 2010; 328(5986): 1705-1709.

[28] Atarashi K, Tanoue T, Shima T, et al. Induction of colonic regulatory $\mathrm{T}$ cells by indigenous Clostridium species. Science. 2011; 331(6015): 337-341.

[29] Ahern PP, Maloy KJ. Understanding immune-microbiota interactions in the intestine. Immunology. 2020; 159(1): 4-14.

[30] Wu C, Sartor RB, Huang K, et al. Transient activation of mucosal effector immune responses by resident intestinal bacteria in normal hosts is regulated by interleukin-10 signalling. Immunology. 2016; 148(3): 304-314.

[31] Zurita-Turk M, Mendes Souza B, Prosperi de Castro C, et al. Attenuation of intestinal inflammation in IL-10 deficient mice by a plasmid carrying Lactococcus lactis strain. BMC Biotechnol. 2020; 20(1): 38. 
[32] Mishima Y, Oka A, Liu B, et al. Microbiota maintain colonic homeostasis by activating TLR2/MyD88/PI3K signaling in IL-10-producing regulatory B cells. J Clin Invest. 2019; 129(9): 3702-3716.

[33] Zhao Y, Chen F, Wu W, et al. GPR43 mediates microbiota metabolite SCFA regulation of antimicrobial peptide expression in intestinal epithelial cells via activation of mTOR and STAT3. Mucosal Immunol. 2018; 11(3): 752-762.

[34] Sun M, Wu W, Chen L, et al. Microbiota-derived short-chain fatty acids promote Th1 cell IL-10 production to maintain intestinal homeostasis. Nat Commun. 2018; 9(1): 3555.

[35] Ramakrishna C, Kujawski M, Chu H, et al. Bacteroides fragilis polysaccharide $A$ induces IL-10 secreting $B$ and $T$ cells that prevent viral encephalitis. Nat Commun. 2019; 10(1): 2153.

[36] Quevrain E, Maubert MA, Michon C, et al. Identification of an anti-inflammatory protein from Faecalibacterium prausnitzii, a commensal bacterium deficient in Crohn's disease. Gut. 2016; 65(3): 415-425.

[37] Lopez-Siles M, Duncan SH, Garcia-Gil LJ, et al. Faecalibacterium prausnitzii: from microbiology to diagnostics and prognostics. ISME J. 2017; 11(4): 841-852.

[38] Xu J, Liang R, Zhang W, et al. Faecalibacterium prausnitziiderived microbial anti-inflammatory molecule regulates intestinal integrity in diabetes mellitus mice via modulating tight junction protein expression. J Diabetes. 2020; 12(3): 224-236.

[39] Arumugam M, Raes J, Pelletier E, et al. Enterotypes of the human gut microbiome. Nature. 2011;473(7346): 174-180.

[40] David LA, Maurice CF, Carmody RN, et al. Diet rapidly and reproducibly alters the human gut microbiome. Nature. 2014; 505(7484): 559-563.

[41] DeGruttola AK, Low D, Mizoguchi A, et al. Current Understanding of Dysbiosis in Disease in Human and Animal Models. Inflamm Bowel Dis. 2016; 22(5): 11371150.

[42] Weiss GA, Hennet T. Mechanisms and consequences of intestinal dysbiosis. Cell Mol Life Sci. 2017; 74(16): 29592977.

[43] Wen L, Duffy A. Factors Influencing the Gut Microbiota, Inflammation, and Type 2 Diabetes. J Nutr. 2017; 147(7): 1468-1475.

[44] Dahmus JD, Kotler DL, Kastenberg DM, et al. The gut microbiome and colorectal cancer: a review of bacterial pathogenesis. J Gastrointest Oncol. 2018; 9(4): 769-777.

[45] Shin W, Kim HJ. Intestinal barrier dysfunction orchestrates the onset of inflammatory host-microbiome cross-talk in a human gut inflammation-on-a-chip. Proc Natl Acad Sci. 2018; 115(45): 10539-10547.

[46] Ahluwalia B, Moraes L, Magnusson MK, et al. Immunopathogenesis of inflammatory bowel disease and mechanisms of biological therapies. Scand J Gastroenterol. 2018; 53(4): 379-389.
[47] Mishima Y, Sartor RB. Manipulating resident microbiota to enhance regulatory immune function to treat inflammatory bowel diseases. J Gastroenterol. 2020; 55(1): 4-14.

[48] Jeon JH, Ahn KB, Kim SK, et al. Bacterial flagellin induces IL-6 expression in human basophils. Mol Immunol. 2015; 65(1): 168-176.

[49] Rolli J, Loukili N, Levrand S, et al. Bacterial flagellin elicits widespread innate immune defense mechanisms, apoptotic signaling, and a sepsis-like systemic inflammatory response in mice. Crit Care. 2010; 14(4): 160.

[50] Shikama Y, Kuroishi T, Nagai Y, et al. Muramyldipeptide augments the actions of lipopolysaccharide in mice by stimulating macrophages to produce pro-IL-1 beta and by down-regulation of the suppressor of cytokine signaling 1 (SOCS1). Innate Immun. 2011; 17(1) :3-15.

[51] Kaneko N, Kurata $M$, Yamamoto $T$, et al. The role of interleukin-1 in general pathology. Inflamm Regen. 2019; (39): 12.

[52] Mailer RK, Joly AL, Liu S, et al. IL-1beta promotes Th17 differentiation by inducing alternative splicing of FOXP3. Sci Rep. 2015; (5): 14674.

[53] Lin YD, Arora J, Diehl K, et al. Vitamin D Is Required for ILC3 Derived IL-22 and Protection From Citrobacter rodentium Infection. Front Immunol. 2019; (10): 1.

[54] Zeng B, Shi S, Ashworth G, et al. ILC3 function as a doubleedged sword in inflammatory bowel diseases. Cell Death Dis. 2019; 10(4): 315.

[55] Hersoug LG, Moller P, Loft S. Role of microbiota-derived lipopolysaccharide in adipose tissue inflammation, adipocyte size and pyroptosis during obesity. Nutr Res Rev. 2018; 31(2): 153-163.

[56] Knox NC, Forbes JD, Peterson CL, et al. The Gut Microbiome in Inflammatory Bowel Disease: Lessons Learned From Other Immune-Mediated Inflammatory Diseases. Am J Gastroenterol. 2019; 114(7): 1051-1070.

[57] Zhu W, Winter MG, Byndloss MX, et al. Precision editing of the gut microbiota ameliorates colitis. Nature. 2018; 553(7687): 208-211.

[58] Khan I, Ullah N, Zha L, et al. Alteration of Gut Microbiota in Inflammatory Bowel Disease (IBD): Cause or Consequence? IBD Treatment Targeting the Gut Microbiome. Pathogens. 2019; 8(3): 126.

[59] Grivennikov SI, Wang K, Mucida D, et al. Adenoma-linked barrier defects and microbial products drive IL-23/IL-17mediated tumour growth. Nature. 2012; 491(7423): 254258.

[60] Miro-Blanch J, Yanes O. Epigenetic Regulation at the Interplay Between Gut Microbiota and Host Metabolism. Front Genet. 2019; (10): 638.

[61] Corte V, Andrade AC, Diaz-Bulnes P, et al. Microbiota derived short chain fatty acids, propionate and butyrate, contribute to modulate the inflammatory response in chronic kidney disease. Nephrol Dial Transplant. 2020; 35(Suppl. 3): 147. 